\title{
Correction to: Extraction of Nano Cellulose Fibres and Their Eco-friendly Polymer Composite
}

\author{
Bashiru Kayode Sodipo and Folahan Abdul Wahab Taiwo Owolabi
}

\section{Correction to:}

Chapter "Extraction of Nano Cellulose Fibres and Their Eco-friendly Polymer Composite" in: Inamuddin et al. (eds.), Sustainable Polymer Composites and Nanocomposites, https://doi.org/10.1007/978-3-030-05399-4_8

The original version of this chapter was inadvertently published with the incorrect author sequence and corresponding author. This has now been corrected. 